\title{
A modified Perry's conjugate gradient method-based derivative-free method for solving large-scale nonlinear monotone equations *
}

\author{
Zhifeng Dai ${ }^{1,2}$, Xiaohong Chen ${ }^{2}$, Fenghua $\mathrm{Wen}^{2 \dagger}$ \\ ${ }^{1}$ College of Mathematics and Computational Science, Changsha University of Science and \\ Technology, Hunan, 410114, China \\ ${ }^{2}$ College of business, Central South University, Hunan, 410083, China.
}

\begin{abstract}
In this paper, we propose a derivative-free method for solving large-scale nonlinear monotone equations. It combines the modified Perry's conjugate gradient method [19] for unconstrained optimization problems and the hyperplane projection method [8]. We prove that the proposed method converges globally if the equations are monotone and Lipschitz continuous without differentiability requirement on the equations, which makes it possible to solve some nonsmooth equations. Another good property of the proposed method is that it is suitable to solve large-scale nonlinear monotone equations due to its lower storage requirement. Preliminary numerical results show that the proposed method is promising.
\end{abstract}

Keywords: Monotone equations; Derivative-free method; Global convergence; Projection method. Mathematics Subject Classification (2000) 65H10, 90C25.

\section{Introduction}

In this paper, we consider solving the following nonlinear equations:

$$
F(x)=0,
$$

where $F: R^{n} \longrightarrow R^{n}$ is continuous and monotone. By monotonicity, we mean

$$
\langle F(x)-F(y), x-y\rangle \geq 0, \quad \forall x, y \in R^{n} .
$$

Nonlinear monotone equations arise in various applications such as subproblems in the generalized proximal algorithms with Bregman distances [1]. Some monotone variational inequality problems can also be converted into systems of nonlinear monotone equations by means of fixed point maps or normal maps if the underlying function satisfies some coercive conditions [2].

Among the iterative methods for solving problem (1.1), the Newton method, quasi-Newton methods, and their variants are attractive because of their fast local convergence property (see

${ }^{*}$ This work is supported by the NSF of China granted 11301041, 11371154.

${ }^{\dagger}$ The corresponding author: wfh@amss.ac.cn 
$[3,4,5],[6])$. The main drawback of these methods, for large values of $\mathrm{n}$, is that they need to solve a linear system of equations at each iteration using the Jacobian matrix or an approximation of it.

In recent years, great efforts have been made to find a solution of problem (1.1) especially for large-scale nonlinear monotone equations. Zhang and Zhou [9] combined the spectral gradient method [7] with the projection method [8] to solve nonlinear monotone equations. The method in [9] is globally convergent if the nonlinear equations are Lipschitz continuous. In addition, Yu et al. [11] proposed a spectral gradient procedure and a projection method, respectively, for nonlinear monotone equations with convex constraints.

Recently, researchers also aim at developing conjugate gradient based method for solving largescale nonlinear equations. Cheng [22] first introduced a PRP-type method for nonlinear monotone equations, which is a combination of the well-known PRP conjugate gradient method and the hyperplane projection method [8]. Then some derivative-free methods were presented which also belong to the conjugate gradient scheme. $\mathrm{Li}$ and $\mathrm{Li}[17]$ proposed a three-term PRP based [13] derivative-free iterative method for solving the nonlinear monotone equations (1.1). Combined the three-term HS [14] methods with the projection scheme in Solodov \& Svaiter [8], Yan et al. [18] proposed a derivative-free iterative method for solving the nonlinear monotone equations (1.1). In addition, Li [17] and Yan et al.[18] discussed the two-term version derivative-free method in Cheng [21] for solving the nonlinear monotone equations (1.1). Xiao and Zhu [15] presented a modified version of the CG_DESCENT method of Hager and Zhang [12] to solve the constrained nonlinear monotone equations. And under some mild conditions, they proved that their proposed method is globally convergent. More recently, Li [25] extended the modified Liu-Storey method [24] to solve the nonlinear monotone equations.

The conjugate gradient-based derivative-free methods for nonlinear monotone equations possess some nice properties. First, due to the lower storage requirement, they are suitable for solving large-scale equations. Second, the methods are function value-based methods and can be applied to solve nonsmooth equations. Third, global convergence is achieved without the differentiability assumption on the equations. In addition, the methods are globally convergent even when the solution set is not a singleton.

In this paper, we will develop an efficient derivative-free algorithm for solving nonlinear monotone equations (1.1). Our work can be considered as a further research of the modified Perry's conjugate gradient method [19] in unconstrained optimization. Here, we extend it to solve nonlinear monotone equations with some little modifications. The rest of this paper is organized as follows. In Section 2, we first propose the method. In Section 3, we prove the global convergence of the proposed method. In Section 4, we report some numerical results to test the proposed method. 


\section{Motivation and the algorithm}

In this section, we simply introduce modified Perry's conjugate gradient method in [19] for solving large-scale unconstrained optimization problems and describe the hyperplane projection method of Solodov and Svaiter. Combined with the hyperplane projection method, we extend modified Perry's conjugate gradient method [19] to solve large-scale constrained nonlinear equations (1.1).

In [19], Livieris and Pintelas proposed a modified Perry's conjugate gradient method. In order to guarantee that the proposed method generates descent directions, Livieris and Pintelas [19] exploit the idea of the spectral-type modified Fletcher-Reeves method $[9,10]$ to the well-known Perry's conjugate gradient method[20]. Under suitable conditions, they establish the global convergence of our proposed method provided that the line search satisfy the Wolfe conditions. Numerical results demonstrate that the proposed method is promising.

Motivated by the modified Perry's conjugate gradient method [19] as well as by the projection scheme in Solodov \& Svaiter [8], we propose an efficient derivative-free algorithm for solving nonlinear monotone equations (1.1). Firstly, we define the search direction as follows

$$
d_{k}= \begin{cases}-F_{0}, & \text { if } \quad k=0, \\ -\left(I+\beta_{k}^{\mathrm{MP}} \frac{F_{k}^{T} d_{k-1}}{\left\|F_{k}\right\|^{2}}\right) F_{k}+\beta_{k}^{\mathrm{MP}} d_{k-1}, & \text { if } \quad k \geq 1,\end{cases}
$$

where

$$
\begin{gathered}
\beta_{k}^{\mathrm{MP}}=\frac{F_{k}^{T}\left(y_{k-1}-\bar{s}_{k-1}\right)}{d_{k-1}^{T} w_{k-1}} \\
w_{k-1}=y_{k-1}+\gamma \bar{s}_{k-1}, \gamma>0, \quad y_{k-1}=F\left(z_{k-1}\right)-F\left(x_{k-1}\right), \quad \bar{s}_{k-1}=z_{k-1}-x_{k-1}=\alpha_{k-1} d_{k-1} .
\end{gathered}
$$

For convenience, we call the method (2.1) and (2.2) as MPD method.

It is easy to see that the condition

$$
F_{k}^{T} d_{k}=-\left\|F_{k}\right\|^{2}
$$

holds, for any line search.

About the hyperplane projection method in [8], note that by the monotonicity of $F$, for any $\tilde{x}$ such that $F(\tilde{x})=0$, we have

$$
\left\langle F\left(z_{k}\right), \tilde{x}-z_{k}\right\rangle \leq 0
$$

Let $x_{k}$ be the current iterate. By performing some kind of line search procedure along a direction $d_{k}$, a point $z_{k}=x_{k}+\alpha_{k} \bar{d}_{k}$ can be computed such that

$$
\left\langle F\left(z_{k}\right), x_{k}-z_{k}\right\rangle>0
$$

Thus the hyperplane

$$
H_{k}=\left\{x \in R^{n} \mid\left\langle F\left(z_{k}\right), x-z_{k}\right\rangle=0\right\} .
$$


strictly separates the current iterate $x_{k}$ from the zeros of equation (1.1). Therefore, it is reasonable to let the next iterate $x_{k+1}$ be the projection of $x_{k}$ onto the hyperplane. Specifically, $x_{k+1}$ is determined by

$$
x_{k+1}=x_{k}-\frac{F\left(z_{k}\right)^{T}\left(x_{k}-z_{k}\right)}{\left\|F\left(z_{k}\right)\right\|^{2}} F\left(z_{k}\right) .
$$

In the remainder of this paper, we always assume that $\mathrm{F}$ satisfies the following assumptions.

\section{Assumption (A)}

(i) Function $F$ is monotone:

$$
\langle F(x)-F(y), x-y\rangle \geq 0, \quad \forall x, y \in R^{n} .
$$

(ii) Function $F$ is Lipschitz continuous, i,e., there is a constant $L>0$ such that

$$
\|F(x)-F(y)\| \leq L\|x-y\|, \quad \forall x, y \in R^{n} .
$$

We state the steps of the algorithm as follows.

\section{Algorithm 2.1.}

Step 0: Given an initial point $x_{0} \in R^{n}$, and constants $\beta>0, \sigma>0, \rho \in(0,1)$. Let $\mathrm{k}:=0$.

Step 1: Stop if $F\left(x_{k}\right)=0$. Otherwise, compute $d_{k}$ by equation (2.1), where $\beta_{k}$ is computed by $(2.2)$ and $(2.3)$.

Step 2: Let $\alpha_{k}=\max \left\{\beta \rho^{i}: i=0,1, \ldots,\right\}$ be determined by the line search rule

$$
-F\left(x_{k}+\alpha_{k} d_{k}\right)^{T} d_{k} \geq \sigma \alpha_{k}\left\|F\left(x_{k}+\alpha_{k} d_{k}\right)\right\| \cdot\left\|d_{k}\right\|^{2} .
$$

Step 3: Compute $z_{k}=x_{k}+\alpha_{k} d_{k}$.

Step 4: Stop if $F\left(z_{k}\right)=0$. Otherwise, update $x_{k+1}$ by (2.5). Let $\mathrm{k}:=\mathrm{k}+1$ and go to Step 1 .

\section{Global convergence analysis}

Lemma 3.1 Let sequences $x_{k}$ and $z_{k}$ be generated by Algorithm 2.1. Then, we have

$$
\alpha_{k} \geq \min \left\{\beta, \frac{\rho}{L+\sigma\left\|F\left(z_{k}^{\prime}\right)\right\|} \frac{\left\|F_{k}\right\|^{2}}{\left\|d_{k}\right\|^{2}}\right\}
$$

where $z_{k}^{\prime}=x_{k}+\alpha_{k}^{\prime} d_{k}, \alpha_{k}^{\prime}=\rho^{-1} \alpha_{k}, \delta=1-\frac{1}{4 \mu}$.

Proof. If $\alpha_{k} \neq \beta$, by the line search process, we have that $\alpha_{k}^{\prime}=\rho^{-1} \alpha_{k}$ does not satisfy (2.8). That is,

$$
-F\left(z_{k}^{\prime}\right)^{T} d_{k}<\sigma \alpha_{k}^{\prime}\left\|F\left(z_{k}^{\prime}\right)\right\| \cdot\left\|d_{k}\right\|^{2} .
$$

It follows from (2.4) and the Lipschitz continuity of $F$ that

$$
\begin{aligned}
\left\|F_{k}\right\|^{2} & =-F_{k}^{T} d_{k}=\left(F\left(z_{k}^{\prime}\right)-F\left(x_{k}\right)\right)^{T} d_{k}-F\left(z_{k}^{\prime}\right)^{T} d_{k} \\
& \leq L \alpha_{k}^{\prime}\left\|d_{k}\right\|^{2}+\sigma \alpha_{k}^{\prime}\left\|F\left(z_{k}^{\prime}\right)\right\| \cdot\left\|d_{k}\right\|^{2} \\
& \leq \rho^{-1} \alpha_{k}\left(L+\sigma\left\|F\left(z_{k}^{\prime}\right)\right\|\right)\left\|d_{k}\right\|^{2} .
\end{aligned}
$$


Hence, we have

$$
\alpha_{k} \geq \frac{\rho}{L+\sigma\left\|F\left(z_{k}^{\prime}\right)\right\|} \frac{\left\|F_{k}\right\|^{2}}{\left\|d_{k}\right\|^{2}}
$$

This implies (3.1).

The next lemma comes from Lemma 2.1 in Solodov \& Svaiter [8], which also holds for Algorithm 2.1. The proof is similar to that in Solodov \& Svaiter [8], and we therefore omit it.

Lemma 3.2 For any $\bar{x}$ satisfying $F(\bar{x})=0$, we have that

$$
\left\|x_{k+1}-\bar{x}\right\|^{2} \leq\left\|x_{k}-\bar{x}\right\|^{2}-\left\|x_{k+1}-x_{k}\right\|^{2} .
$$

In particular, the sequence $\left\{x_{k}\right\}$ is bounded and

$$
\sum_{k=0}^{\infty}\left\|x_{k+1}-x_{k}\right\|^{2}<\infty
$$

Remark 1. Lemma 3.2 reveals an interesting property of Algorithm 2.1, which is that the distance from the iterates to the solution set of equation (1.1) decreases with $\mathrm{k}$.

Lemma 3.3 Suppose that $\left\{x_{k}\right\}$ is generated by Algorithm 2.1, Then, we have

$$
\lim _{k \rightarrow \infty} \alpha_{k}\left\|d_{k}\right\|=0
$$

Proof. For any $k$, it follows from (2.5) and (2.8) that

$$
\left\|x_{k+1}-x_{k}\right\|=\frac{\left|F\left(z_{k}\right)^{T}\left(x_{k}-z_{k}\right)\right|}{\left\|F\left(z_{k}\right)\right\|}=\frac{-\alpha_{k} F\left(z_{k}\right)^{T} d_{k}}{\left\|F\left(z_{k}\right)\right\|} \geq \sigma \alpha_{k}^{2}\left\|d_{k}\right\|^{2} .
$$

In particular, we have that

$$
\lim _{k \rightarrow \infty} \alpha_{k}\left\|d_{k}\right\|=0
$$

The next lemma comes from Lemma 2.4 in Yan et al. [18], which also holds for Algorithm 2.1. Here we omit it.

Lemma 3.4 Suppose that $\left\{x_{k}\right\}$ is generated by Algorithm 2.1, $\bar{x}$ satisfy $F(\bar{x})=0, z_{k}^{\prime}=x_{k}+$ $\alpha_{k}^{\prime} d_{k}, \alpha_{k}^{\prime}=\rho^{-1} \alpha_{k}$. Then, the sequences $\left\{\left\|F_{k}\right\|\right\}$ and $\left\{\left\|F\left(z_{k}^{\prime}\right)\right\|\right\}$ are bounded. That is, there exists constant $M \geq 0$, such that

$$
\left\|F_{k}\right\| \leq M, \quad\left\|F\left(z_{k}^{\prime}\right)\right\| \leq M
$$

The following theorem establishes the global convergence of our method.

Theorem 3.1 Let $\left\{x_{k}\right\}$ be generated by Algorithm 2.1. Then, we have

$$
\liminf _{k \rightarrow \infty}\left\|F_{k}\right\|=0
$$


Proof. For the sake of contradiction, we suppose that the conclusion is not true. Then there exists a constant $\varepsilon>0$ such that $\left\|F_{k}\right\|>\varepsilon$. Since $F_{k} \neq 0$ and with (2.4), it follows that $d_{k} \neq 0$. By the monotonicity of $F$ and (2.1), we have,

$$
\begin{aligned}
\bar{s}_{k-1}^{T} w_{k-1} & =\left\langle F\left(z_{k-1}\right)-F\left(x_{k-1}\right), z_{k-1}-x_{k-1}\right\rangle+\gamma \bar{s}_{k-1}^{T} \bar{s}_{k-1} \\
& \geq \gamma \bar{s}_{k-1}^{T} \bar{s}_{k-1} .
\end{aligned}
$$

This together with the definition of $\bar{s}_{k-1}$ implies

$$
d_{k-1}^{T} w_{k-1} \geq \gamma \alpha_{k-1}\left\|d_{k-1}\right\|^{2}
$$

We have from (2.2), (2.3) and (3.6) that

$$
\begin{aligned}
\left|\beta_{k}^{\mathrm{MP}}\right| & =\left|\frac{F_{k}^{T}\left(y_{k-1}-\bar{s}_{k-1}\right)}{d_{k-1}^{T} w_{k-1}}\right| \leq \frac{\left|F_{k}^{T} y_{k-1}\right|+\left|F_{k}^{T} \bar{s}_{k-1}\right|}{d_{k-1}^{T} w_{k-1}} \\
& \leq \frac{L \alpha_{k-1}\left\|d_{k-1}\right\|\left\|F_{k}\right\|+\alpha_{k-1}\left\|d_{k-1}\right\|\left\|F_{k}\right\|}{\gamma \alpha_{k-1}\left\|d_{k-1}\right\|^{2}} \\
& =\left(\frac{L+1}{\gamma}\right) \frac{\left\|F_{k}\right\|}{\left\|d_{k-1}\right\|} .
\end{aligned}
$$

Therefore, from (2.1) and (3.4), we obtain

$$
\begin{aligned}
\left\|d_{k}\right\| & \leq\left\|F_{k}\right\|+\left|\beta_{k}^{\mathrm{MP}}\right| \frac{\left|F_{k}^{T} d_{k-1}\right|}{\left\|F_{k}\right\|^{2}}\left\|F_{k}\right\|+\left|\beta_{k}^{\mathrm{MP}}\right|\left\|d_{k-1}\right\| \\
& \leq\left\|F_{k}\right\|+2\left(\frac{L+1}{\gamma}\right)\left\|F_{k}\right\| \\
& =\left(1+\frac{2(L+1)}{\gamma}\right) M .
\end{aligned}
$$

Define $C=\left(1+\frac{2(L+1)}{\gamma}\right)$. We have $\left\|d_{k}\right\| \leq C$.

This together with Lemmas 2.1 and 2.4 and inequalities $\left\|d_{k}\right\| \geq \varepsilon,\left\|F_{k}\right\| \geq \varepsilon$ implies for all $k$ sufficiently large,

$$
\begin{aligned}
\alpha_{k}\left\|d_{k}\right\| & \geq \min \left\{\beta, \frac{\delta \rho}{L+\sigma\left\|F\left(z_{k}^{\prime}\right)\right\|} \frac{\left\|F_{k}\right\|^{2}}{\left\|d_{k}\right\|^{2}}\right\}\left\|d_{k}\right\| \\
& \geq \min \left\{\beta \varepsilon, \frac{\delta \rho \varepsilon^{2}}{(L+\sigma M) \| C}\right\}>0 .
\end{aligned}
$$

The last inequality yields a contradiction with (3.2). Consequently, (3.5) holds. The proof is complete.

\section{Numerical experiments}

This section reports some numerical experiments. We test the MPE method and compare it with the modified-PRP-based derivative-free method in Li and Li[17](called MPRPL) and the modifiedHS-based derivative-free method in Yan [18](called MHSY).

The methods in Tables have the following meanings: 
- "MPD" stands for the modified-Perry's-CG-based derivative-free method with the search direction 2.1

- "MPRPL" stands for the modified-PRP-based derivative-free method with the following search direction

$$
d_{k}= \begin{cases}-F_{0}, & \text { if } \quad k=0, \\ -\left(I+\beta_{k} \mathrm{PRP} \frac{F_{k}^{T} d_{k-1}}{\left\|F_{k}\right\|^{2}}\right) F_{k}+\beta_{k}^{\mathrm{PRP}} d_{k-1}, & \text { if } \quad k \geq 1,\end{cases}
$$

where

$$
y_{k-1}=F_{k}-F_{k-1}, \quad \beta_{k}^{\mathrm{PRP}}=\frac{F_{k}^{T} y_{k-1}}{\left\|F_{k-1}\right\|^{2}} .
$$

- "MHSY" stands for the modified-HS-based derivative-free method with the following search direction

$$
d_{k}= \begin{cases}-F_{0}, & \text { if } \quad k=0 \\ -\left(I+\beta_{k}^{\mathrm{MHS}} \frac{F_{k}^{T} d_{k-1}}{\left\|F_{k}\right\|^{2}}\right) F_{k}+\beta_{k}^{\mathrm{MHS}} d_{k-1}, & \text { if } \quad k \geq 1\end{cases}
$$

where

$$
\begin{gathered}
y_{k-1}=F_{k}-F_{k-1}, \quad \beta_{k}^{\mathrm{MHS}}=\frac{F_{k}^{T} w_{k-1}}{d_{k-1}^{T} w_{k-1}}, \quad, w_{k}=y_{k}+t\left\|F_{k}\right\| \bar{s}_{k} \\
\bar{s}_{k}=z_{k}-x_{k}=\alpha_{k} d_{k}, \quad t=1+\left\|F_{k}\right\|^{-1} \max \left(0,-\frac{y_{k}^{T} \bar{s}_{k}}{\left\|\bar{s}_{k}\right\|^{2}}\right) .
\end{gathered}
$$

For the three methods, we choose the same initial trial steplength as

$$
\beta=\left|\frac{F\left(x_{k}\right)^{T} d_{k}}{d_{k}^{T}\left(F\left(x_{k}+\epsilon d_{k}\right)-F\left(x_{k}\right)\right) / \epsilon}\right| \approx\left|\frac{F\left(x_{k}\right)^{T} d_{k}}{d_{k}^{T} \nabla F\left(x_{k}\right) d_{k}}\right|, \quad \epsilon=10^{-8} .
$$

In order to improve the efficiency of the three methods in practical computation, we utilize the following line search process.

\section{Line Search Process:}

If inequality

$$
-F\left(x_{k}+\beta d_{k}\right)^{T} d_{k} \geq \sigma \beta\left\|F\left(x_{k}+\beta d_{k}\right)\right\| \cdot\left\|d_{k}\right\|^{2},
$$

holds, then we let $\alpha_{k}=\beta$. Otherwise we let $\alpha_{k}$ be the largest scalar in the set $\left\{\beta \rho^{i}, i=0,1, \ldots\right\}$ such that inequality (4.4) is satisfied. In addition, if the above $\beta$ satisfies $\beta<10^{-4}$, we set $\beta=1$. In the line search process, $\rho=0.5, \sigma=2$.

We stop the iteration if the condition $\min \left\{\left\|F\left(x_{k}\right)\right\|,\left\|F\left(z_{k}\right)\right\|\right\} \leq$ atol + rtol $\left\|F\left(x_{0}\right)\right\|$ is satisfied, where $a t o l=r t o l=10^{-4}$. All of the algorithms are coded in Matlab 7.0 and run on a personal computer with a 2.0 GHZ CPU processor.

We test the performance of MPD, MPRPL, MHSY methods on the following five problems with different initial points and various sizes .

Problem 1([23]). The elements of function $F(x)$ are given by

$$
\begin{aligned}
& F_{1}(x)=2 x_{1}+\sin \left(x_{1}\right)-1, \\
& F_{i}(x)=-2 x_{i-1}+2 x_{i}+\sin \left(x_{i}\right)-1, \quad i=2, \ldots, n-1, \\
& F_{n}(x)=2 x_{n}+\sin \left(x_{n}\right)-1 .
\end{aligned}
$$


Problem 2 ([6]). The elements of $F(x)$ are given by

$$
F_{i}(x)=x_{i}-\sin \left(x_{i}\right), \quad i=1, \ldots, n .
$$

Problem 3 ([23]). The elements of $F(x)$ are given by

$$
F_{i}(x)=2 x_{i}-\sin \left(\left|x_{i}\right|\right), \quad i=1, \ldots, n .
$$

Problem 4 ([6]). Function $F(x)$ is given by

$$
F(x)=\mathrm{A} x+g(x)
$$

where $g(x)=\left(e^{x_{1}}-1, e^{x_{2}}-1, \ldots, e^{x_{n}}-1\right)^{T}$

$$
\mathrm{A}=\left(\begin{array}{ccccc}
2 & -1 & & & \\
-1 & 2 & -1 & & \\
& \ddots & \ddots & \ddots & \\
& & \ddots & \ddots & -1 \\
& & & -1 & 2
\end{array}\right)
$$

Problem 5. Function $F(x)$ is given by

$$
F(x)=\mathrm{A} x+|\mathrm{X}|-\mathrm{B}
$$

where $|\mathrm{X}|=\left(\left|x_{1}\right|,\left|x_{2}\right|, \ldots,\left|x_{n}\right|\right)^{T}, \mathrm{~B}=(1,1, \ldots, 1)^{T}$, and

$$
A=\left(\begin{array}{ccccc}
2 & -1 & & & \\
-1 & 2 & -1 & & \\
& \ddots & \ddots & \ddots & \\
& & \ddots & \ddots & -1 \\
& & & -1 & 2
\end{array}\right)
$$

The results are listed in Tables $1-5$, where $x_{1}=(0.1, \ldots, 0.1)^{T}, x_{2}=(1, \ldots, 1)^{T}, x_{3}=\left(1, \frac{1}{2}\right.$, $\left.\ldots, \frac{1}{n}\right)^{T}, x_{4}=(-10, \ldots,-10)^{T}, x_{5}=(-0.1, \ldots,-0.1)^{T}, x_{6}=(-1, \ldots,-1)^{T}$. We will report the following results: the CPU time Time (in seconds), the number of iterations Iter, and the number of function evaluations Feval. 
Table 1: Test results for Problem 1 with given initial points.

\begin{tabular}{|c|c|c|c|c|c|c|c|c|c|c|}
\hline \multirow[t]{2}{*}{ Initial } & \multirow[t]{2}{*}{$\mathrm{n}$} & \multicolumn{3}{|c|}{ MPRPL } & \multicolumn{3}{|c|}{ MHSY } & \multicolumn{3}{|c|}{ MPD } \\
\hline & & Time & Iter & Feval & Time & Iter & Feval & Time & Iter & Feval \\
\hline$x_{1}$ & 1000 & 0.17 & 196 & 591 & 0.19 & 198 & 646 & 0.12 & 190 & 580 \\
\hline$x_{2}$ & 1000 & 0.11 & 203 & 612 & 0.14 & 204 & 624 & 0.17 & 192 & 600 \\
\hline$x_{3}$ & 1000 & 0.19 & 195 & 588 & 0.24 & 193 & 201 & 0.17 & 192 & 582 \\
\hline$x_{4}$ & 1000 & 0.15 & 243 & 741 & 0.18 & 256 & 762 & 0.14 & 230 & 709 \\
\hline$x_{5}$ & 1000 & 0.17 & 194 & 585 & 0.19 & 209 & 688 & 0.18 & 190 & 572 \\
\hline$x_{6}$ & 1000 & 0.16 & 189 & 571 & 0.25 & 231 & 657 & 0.15 & 182 & 550 \\
\hline$x_{1}$ & 5000 & 8.19 & 487 & 1465 & 8.38 & 523 & 1547 & 8.09 & 468 & 1428 \\
\hline$x_{2}$ & 5000 & 12.85 & 769 & 2310 & 13.87 & 823 & 2722 & 12.53 & 744 & 2255 \\
\hline$x_{3}$ & 5000 & 7.98 & 469 & 1411 & 9.01 & 524 & 1849 & 7.68 & 449 & 1371 \\
\hline$x_{4}$ & 5000 & 15.96 & 929 & 2840 & 18.74 & 1000 & 3261 & 15.61 & 900 & 2771 \\
\hline$x_{5}$ & 5000 & 7.59 & 453 & 1363 & 8.05 & 526 & 1653 & 7.29 & 432 & 1320 \\
\hline$x_{6}$ & 5000 & 6.32 & 371 & 1122 & 7.32 & 436 & 1476 & 6.02 & 348 & 1073 \\
\hline$\overline{x_{1}}$ & 10000 & 34.59 & 539 & 1622 & 37.90 & 600 & 2002 & 10.31 & 530 & 1605 \\
\hline$x_{2}$ & 10000 & 65.06 & 1023 & 3072 & 72.6 & 1054 & 3166 & 67.79 & 1017 & 3054 \\
\hline$x_{3}$ & 10000 & 34.30 & 516 & 1553 & 35.99 & 533 & 1569 & 35.03 & 516 & 1554 \\
\hline$x_{4}$ & 10000 & 109.65 & 1668 & 5075 & 116.8 & 1742 & 5668 & 109.65 & 1668 & 5075 \\
\hline$x_{5}$ & 10000 & 31.57 & 497 & 1497 & 33.63 & 532 & 1568 & 32.02 & 495 & 1491 \\
\hline$x_{6}$ & 10000 & 25.36 & 396 & 1202 & 26.70 & 405 & 1206 & 26.11 & 394 & 1196 \\
\hline
\end{tabular}

Table 2: Test results for Problem 2 with given initial points.

\begin{tabular}{|c|c|c|c|c|c|c|c|c|c|c|}
\hline \multirow[t]{2}{*}{ Initial } & \multirow[t]{2}{*}{$\mathrm{n}$} & \multicolumn{3}{|c|}{ MPRPL } & \multicolumn{3}{|c|}{ MHSY } & \multicolumn{3}{|c|}{ MPD } \\
\hline & & Time & Iter & Feval & Time & Iter & Feval & Time & Iter & Feval \\
\hline$x_{1}$ & 1000 & 0.81 & 12 & 39 & 1.15 & 17 & 36 & 0.62 & 12 & 27 \\
\hline$x_{2}$ & 1000 & 0.81 & 12 & 39 & 1.15 & 17 & 36 & 0.62 & 12 & 27 \\
\hline$x_{3}$ & 1000 & 0.55 & 8 & 27 & 0.75 & 11 & 24 & 0.45 & 8 & 19 \\
\hline$x_{4}$ & 1000 & 0.85 & 9 & 42 & 1.20 & 14 & 44 & 0.71 & 9 & 33 \\
\hline$x_{5}$ & 1000 & 0.53 & 6 & 28 & 1.14 & 13 & 42 & 0.45 & 6 & 22 \\
\hline$x_{6}$ & 1000 & 0.53 & 6 & 28 & 1.14 & 13 & 42 & 0.45 & 6 & 22 \\
\hline$x_{1}$ & 5000 & 5.32 & 13 & 42 & 6.93 & 17 & 36 & 3.90 & 13 & 29 \\
\hline$x_{2}$ & 5000 & 5.01 & 12 & 39 & 6.93 & 17 & 36 & 3.65 & 12 & 27 \\
\hline$x_{3}$ & 5000 & 3.01 & 7 & 24 & 4.19 & 10 & 22 & 2.23 & 7 & 17 \\
\hline$x_{4}$ & 5000 & 7.38 & 12 & 60 & 10.42 & 19 & 64 & 5.99 & 12 & 48 \\
\hline$x_{5}$ & 5000 & 3.40 & 6 & 28 & 6.94 & 13 & 42 & 2.74 & 6 & 22 \\
\hline$x_{6}$ & 5000 & 3.40 & 6 & 28 & 6.94 & 13 & 42 & 2.74 & 6 & 22 \\
\hline$x_{1}$ & 10000 & 21.15 & 13 & 42 & 27.57 & 17 & 36 & 15.61 & 13 & 29 \\
\hline$x_{2}$ & 10000 & 19.65 & 12 & 39 & 27.58 & 17 & 36 & 14.36 & 12 & 27 \\
\hline$x_{3}$ & 10000 & 11.92 & 7 & 24 & 14.94 & 9 & 20 & 8.91 & 7 & 17 \\
\hline$x_{4}$ & 10000 & 35.15 & 14 & 72 & 44.61 & 20 & 69 & 28.98 & 14 & 58 \\
\hline$x_{5}$ & 10000 & 13.86 & 6 & 28 & 29.46 & 14 & 45 & 11.06 & 6 & 22 \\
\hline$x_{6}$ & 10000 & 13.86 & 6 & 28 & 29.46 & 14 & 45 & 11.06 & 6 & 22 \\
\hline
\end{tabular}


Table 3 Test results for Problem 3 with given initial points.

\begin{tabular}{|c|c|c|c|c|c|c|c|c|c|c|}
\hline \multirow[t]{2}{*}{ Initial } & \multirow[t]{2}{*}{$\mathrm{n}$} & \multicolumn{3}{|c|}{ MPRPL } & \multicolumn{3}{|c|}{ MHSY } & \multicolumn{3}{|c|}{ MPD } \\
\hline & & Time & Iter & Feval & Time & Iter & Feval & Time & Iter & Feval \\
\hline$x_{1}$ & 1000 & 0.81 & 12 & 39 & 1.15 & 17 & 36 & 0.62 & 12 & 27 \\
\hline$x_{2}$ & 1000 & 0.81 & 12 & 39 & 1.15 & 17 & 36 & 0.62 & 12 & 27 \\
\hline$x_{3}$ & 1000 & 0.55 & 8 & 27 & 0.75 & 11 & 24 & 0.45 & 8 & 19 \\
\hline$x_{4}$ & 1000 & 0.85 & 9 & 42 & 1.20 & 14 & 44 & 0.71 & 9 & 33 \\
\hline$x_{5}$ & 1000 & 0.53 & 6 & 28 & 1.14 & 13 & 42 & 0.45 & 6 & 22 \\
\hline$x_{6}$ & 1000 & 0.53 & 6 & 28 & 1.14 & 13 & 42 & 0.45 & 6 & 22 \\
\hline$x_{1}$ & 5000 & 5.32 & 13 & 42 & 6.93 & 17 & 36 & 3.90 & 13 & 29 \\
\hline$x_{2}$ & 5000 & 5.01 & 12 & 39 & 6.93 & 17 & 36 & 3.65 & 12 & 27 \\
\hline$x_{3}$ & 5000 & 3.01 & 7 & 24 & 4.19 & 10 & 22 & 2.23 & 7 & 17 \\
\hline$x_{4}$ & 5000 & 7.38 & 12 & 60 & 10.42 & 19 & 64 & 5.99 & 12 & 48 \\
\hline$x_{5}$ & 5000 & 3.40 & 6 & 28 & 6.94 & 13 & 42 & 2.74 & 6 & 22 \\
\hline$x_{6}$ & 5000 & 3.40 & 6 & 28 & 6.94 & 13 & 42 & 2.74 & 6 & 22 \\
\hline$x_{1}$ & 10000 & 21.15 & 13 & 42 & 27.57 & 17 & 36 & 15.61 & 13 & 29 \\
\hline$x_{2}$ & 10000 & 19.65 & 12 & 39 & 27.58 & 17 & 36 & 14.36 & 12 & 27 \\
\hline$x_{3}$ & 10000 & 11.92 & 7 & 24 & 14.94 & 9 & 20 & 8.91 & 7 & 17 \\
\hline$x_{4}$ & 10000 & 35.15 & 14 & 72 & 44.61 & 20 & 69 & 28.98 & 14 & 58 \\
\hline$x_{5}$ & 10000 & 13.86 & 6 & 28 & 29.46 & 14 & 45 & 11.06 & 6 & 22 \\
\hline$x_{6}$ & 10000 & 13.86 & 6 & 28 & 29.46 & 14 & 45 & 11.06 & 6 & 22 \\
\hline
\end{tabular}

Table 4: Test results for Problem 4 with given initial points.

\begin{tabular}{|c|c|c|c|c|c|c|c|c|c|c|}
\hline \multirow[t]{2}{*}{ Initial } & \multirow[t]{2}{*}{$\mathrm{n}$} & \multicolumn{3}{|c|}{ MPRPL } & \multicolumn{3}{|c|}{ MHSY } & \multicolumn{3}{|c|}{ MPD } \\
\hline & & Time & Iter & Feval & Time & Iter & Feval & Time & Iter & Feval \\
\hline$x_{1}$ & 1000 & 0.06 & 50 & 219 & 0.07 & 59 & 231 & 0.05 & 36 & 163 \\
\hline$x_{2}$ & 1000 & 0.06 & 56 & 252 & 0.07 & 61 & 263 & 0.05 & 36 & 165 \\
\hline$x_{3}$ & 1000 & 0.05 & 34 & 152 & 0.08 & 38 & 175 & 0.03 & 28 & 127 \\
\hline$x_{4}$ & 1000 & 0.07 & 100 & 421 & 0.08 & 113 & 529 & 0.06 & 86 & 379 \\
\hline$x_{5}$ & 1000 & 0.06 & 42 & 187 & 0.08 & 53 & 203 & 0.03 & 37 & 167 \\
\hline$x_{6}$ & 1000 & 0.06 & 60 & 261 & 0.07 & 81 & 288 & 0.03 & 42 & 189 \\
\hline$x_{1}$ & 5000 & 1.11 & 48 & 209 & 1.15 & 63 & 262 & 0.95 & 37 & 163 \\
\hline$x_{2}$ & 5000 & 1.19 & 46 & 224 & 1.45 & 55 & 282 & 0.82 & 31 & 149 \\
\hline$x_{3}$ & 5000 & 0.62 & 27 & 117 & 0.65 & 32 & 126 & 0.65 & 27 & 121 \\
\hline$x_{4}$ & 5000 & 2.59 & 109 & 483 & 2.72 & 116 & 558 & 2.39 & 94 & 435 \\
\hline$x_{5}$ & 5000 & 1.21 & 53 & 231 & 1.86 & 60 & 278 & 0.97 & 40 & 179 \\
\hline$x_{6}$ & 5000 & 1.20 & 54 & 234 & 1.97 & 61 & 275 & 1.06 & 43 & 193 \\
\hline$x_{1}$ & 10000 & 3.96 & 45 & 195 & 4.30 & 78 & 222 & 3.63 & 39 & 175 \\
\hline$x_{2}$ & 10000 & 4.27 & 41 & 210 & 5.26 & 47 & 234 & 2.84 & 28 & 137 \\
\hline$x_{3}$ & 10000 & 1.93 & 22 & 96 & 2.12 & 25 & 105 & 2.32 & 25 & 112 \\
\hline$x_{4}$ & 10000 & 10.86 & 117 & 533 & 11.32 & 123 & 539 & 12.05 & 119 & 594 \\
\hline$x_{5}$ & 10000 & 4.34 & 49 & 212 & 5.06 & 51 & 232 & 3.65 & 40 & 180 \\
\hline$x_{6}$ & 10000 & 4.89 & 56 & 246 & 5.65 & 66 & 253 & 3.75 & 41 & 185 \\
\hline
\end{tabular}


Table 5: Test results for Problem 5 with given initial points.

\begin{tabular}{|c|c|c|c|c|c|c|c|c|c|c|}
\hline \multirow[t]{2}{*}{ Initial } & \multirow[t]{2}{*}{$\mathrm{n}$} & \multicolumn{3}{|c|}{ MPRPL } & \multicolumn{3}{|c|}{ MHSY } & \multicolumn{3}{|c|}{ MPD } \\
\hline & & Time & Iter & Feval & Time & Iter & Feval & Time & Iter & Feval \\
\hline$x_{1}$ & 1000 & 0.66 & 47 & 199 & 0.69 & 49 & 201 & 0.62 & 37 & 165 \\
\hline$x_{2}$ & 1000 & 0.45 & 22 & 105 & 0.48 & 32 & 123 & 0.41 & 21 & 96 \\
\hline$x_{3}$ & 1000 & 0.03 & 48 & 209 & 0.09 & 50 & 275 & 0.05 & 38 & 117 \\
\hline$x_{4}$ & 1000 & 0.47 & 37 & 165 & 0.55 & 49 & 178 & 0.31 & 30 & 94 \\
\hline$x_{5}$ & 1000 & 0.05 & 54 & 237 & 0.06 & 54 & 237 & 0.05 & 41 & 122 \\
\hline$x_{6}$ & 1000 & 0.19 & 274 & 1146 & 0.22 & 325 & 1222 & 0.14 & 155 & 468 \\
\hline$x_{1}$ & 5000 & 1.22 & 51 & 221 & 1.32 & 53 & 229 & 0.92 & 36 & 161 \\
\hline$x_{2}$ & 5000 & 0.56 & 22 & 105 & 0.92 & 28 & 120 & 0.54 & 21 & 96 \\
\hline$x_{3}$ & 5000 & 1.11 & 47 & 200 & 1.80 & 58 & 262 & 0.733 & 38 & 117 \\
\hline$x_{4}$ & 5000 & 1.20 & 48 & 206 & 1.50 & 56 & 224 & 0.72 & 39 & 121 \\
\hline$x_{5}$ & 5000 & 1.17 & 47 & 206 & 2.00 & 56 & 343 & 0.73 & 41 & 119 \\
\hline$x_{6}$ & 5000 & 25.06 & 1108 & 2607 & 28.5 & 1361 & 2781 & 10.45 & 608 & 1826 \\
\hline$x_{1}$ & 10000 & 3.90 & 44 & 191 & 3.92 & 43 & 190 & 3.31 & 36 & 161 \\
\hline$x_{2}$ & 10000 & 51.1 & 22 & 105 & 51.86 & 23 & 110 & 47.1 & 21 & 96 \\
\hline$x_{3}$ & 10000 & 4.24 & 47 & 204 & 5.76 & 50 & 221 & 2.53 & 38 & 117 \\
\hline$x_{4}$ & 10000 & 10.5 & 48 & 209 & 11.20 & 58 & 227 & 6.90 & 40 & 134 \\
\hline$x_{5}$ & 10000 & 4.02 & 45 & 196 & 4.50 & 53 & 221 & 2.48 & 39 & 114 \\
\hline$x_{6}$ & 10000 & 183.1 & 2144 & 3912 & 201.4 & 2040 & 3876 & 76.3 & 1170 & 3511 \\
\hline
\end{tabular}

From tables 1-5, we can see the MPD method performs better than the MPRPL method in Li and Li [17] and obviously better than the MHSY method in Yan et al. [18], which implies that the proposed method is computationally efficient for solving large-scale nonlinear monotone equations.

\section{Conclusions}

In this paper, we have proposed a derivative-free method to solve nonlinear monotone equations which is combined the modified Perry's conjugate gradient method [19] for unconstrained optimization problems and the hyperplane projection method [8]. Under appropriate conditions, we prove that the proposed method converges globally. The proposed method is suitable for large-scale monotone equations because of the low memory requirement. The preliminary numerical results show that our method is promising. In addition, the derivative-free methods can solve absolute value equations, for example Problem 5.

\section{References}

[1] A.N. Iusem, M.V. Solodov. Newton-type methods with generalized distances for constrained optimization. Optimization, 41 (1997) 257-278.

[2] Y.B. Zhao, D.H. Li. Monotonicity of fixed point and normal mapping associated with variational inequality and its application. SIAM Journal on Optimization, 4 (2001) 962-973. 
[3] P.N. Brown, Y. Saad. Convergence theory of nonlinear Newton-Krylov algorithms, SIAM Journal on Optimization, 4 (1994) 297-330.

[4] D.H. Li and M. Fukushima. A globally and superlinear convergent Gauss-Newton based BFGS methods for symmetric nonlinear equations. SIAM Journal on Numerical Analysis, 37 (1999) $152-172$.

[5] D.H. Li and M. Fukushima. A derivative-free line search and global convergence of Broydenlike method for nonlinear equations. Optimization Methods and Software, 13 (2000) 583-599.

[6] W.J. Zhou, D.H. Li. A globally convergent BFGS method for nonlinear monotone equations without any merit functions. Mathematics of Computation, 77 (2008) 2231-2240.

[7] J. Barzilai and J.M. Borwein. Two-point step size gradient methods. IMA Journal on Numerical Analysis, 8 (1988) 141-148.

[8] M.V. Solodov and B.F. Svaiter. A globally convergent inexact Newton method for systems of monotone equations, in: M. Fukushima, L. Qi (Eds.)Reformulation: Nonsmooth, Piecewise Smooth, Semismooth and Smoothing Methods, Kluwer Academic Publishers. (1998) 355-369.

[9] L. Zhang, W. Zhou, D. Li, Global convergence of a modified Fletcher-Reeves conjugate gradient method with Armijo-type line search, Numerische Mathematik, 104 (2006) 561-572.

[10] S.Q. Du, Y.Y. Chen, Global convergence of a modified spectral FR conjugate gradient method, Applied Mathematics and Computation, 202 (2) (2008) 766-770.

[11] Z.S. Yu, L.N Ji, J. Sun, Y.H. Xiao, L.Y. Liu and Z.H. Li. Spectral gradient projection method for monotone nonlinear equations with convex constraints. Applied Numerical Mathematics, 59 (2009) 2416-2423.

[12] W.W. Hager and H. Zhang. A new conjugate gradient method with guaranteed descent and an efficent line search. SIAM Journal on Optimization, 16 (2005) 170-192.

[13] L. Zhang, W.J. Zhou, D.H. Li. A descent modified Polak-Ribiére-Polyak conjugate gradient method and its global convergence. IMA Journal on Numerical Analysis, 26 (2006) 629-640.

[14] L. Zhang, W.J. Zhou, D.H. Li. Some descent three-term conjugate gradient methods and their global convergence. Optimization Methods and Software, 22(2007) 697-711.

[15] Y. Xiao, H. Zhu, A conjugate gradient method to solve convex constrained monotone equations with applications in compressive sensing, Journal of Mathematical Analysis and Applications, 405(2013) 310-319.

[16] L. Zhang, W. Zhou, Spectral gradient projection method for solving nonlinear monotone equations, Journal of Computational and Applied Mathematics, 196 ( 2006) 478-484. 
[17] Q. Li, D.H. Li, A class of derivative-free methods for largescale nonlinearmonotone equations, IMA Journal of Numerical Analysis, 31, (2011) 1625-1635.

[18] Q.R. Yan, X.Z. Peng, D.H. Li. A globally convergent derivative-free method for solving largescale nonlinear monotone equations. Journal of Computational and Applied Mathematics, 234 (2010) 649-657.

[19] I. E. Livieris, P. Pintelas. Globally convergent modified Perrys conjugate gradient method, Applied Mathematics and Computation, 218 (2012) 9197-9207.

[20] A. Perry, A modified conjugate gradient algorithm, Operational Research, 26 (1978) 10731078.

[21] W.Y. Cheng. A two-term PRP-based descent method. Numerical Functional Analysis and optimization, 28 (2007) 1217-1230.

[22] W.Y. Cheng. A PRP type method for systems of monotone equations, Mathematical and Computer Modelling, 50 (2009) 15-20.

[23] W.J. Zhou, D.H. Li . Limited memory BFGS method for nonlinear monotone equations. Journal of Computational Mathematics, 25(2007) 89-96.

[24] M. Li and H. Feng . A sufficient descent Liu-Storey conjugate gradient method for unconstrained optimization problems. Applied Mathematics and Computation, 218 (2011) 15771586.

[25] M. Li, A Liu-Storey type method for solving large-scale nonlinear monotone equations, Numerical Functional Analysis and optimization, 35 (2014) 310-322. 